Es wird vom Empfänger des Preises erwartet, daß er während der Siebenten Jahrestagung der Gesellschaft, vom 15. - 17. September, 1971, in Southampton, England, einen Vortrag über seine Forschungsarbeiten hält und daß er eine zusammenfassende Darstellung zur Publikation in Diabetologia, dem Organ der Gesellschaft, einreicht.

\title{
German Diabetes Association
}

On May 8th 1970, at the 5th Congress of the German Diabetes Association in Bonn - Bad Godesberg the "Ferdinand-Bertram-Preis" and the "Förderpreis der Deutschen Diabetes-Gesellschaft" were presented.

Dr. H.F. Kern, Institute of Anatomy, University of Heidelberg, received the "Ferdinand-Bertram-Preis" for comparative morphological investigations on the islet system of varies species and studies on the ultrastructure of islet cells in animals under various experimental conditions.

The "Förderpreis" was awarded to Dr. Walter Huth, Institute of Physical Chemistry, University of Göttingen, for his thesis "The Mechanism of Gluconeogenesis from Pyruvate and Fumarate".

Veränderungen im Vorstand der Deutschen Diabetes-Gesellschaft:

President 1970/71: Prof. Dr. K. Jahnke, Direktor der Medizinischen Klinik der Ferdinand-Sauerbruch-Krankenanstalten, 56 Wuppertal, Arrenbergstr. 20.

Secretary: Prof. Dr. H. Ditschuneit, Zentrum für Innere Medizin und Kinderheilkunde der Universität Ulm, Ulm/Donau, Steinhövelstr. 9.

The 6th Congress of the German Diabetes Association will take place at Düsseldorf (Hotel Düsseldorf Hilton) on May 14th and 15th, 1971. Papers on all topics in the field of diabetes may be delivered, deadline for announcement being February 10th, 1971. For further information: Prof. Dr. K. Jahnke, Medizinische Klinik, Städtische Krankenanstalten 56 Wuppertal, Arrenbergerstr. 20

\section{Ferdinand-Bertram-Preis 1970}

Der „Ferdinand-Bertram-Preis“" wurde von der Firma Boehringer Mannheim GmbH für hervorragende und wegweisende wissenschaftliche Arbeiten auf dem Gebiet der Diabetologie gestiftet. Er wird von der Deutschen Diabetes-Gesellschaft verliehen und ist mit DMI 5000, - dotiert. - Deutschsprachige Bewerber, die das 40 . Lebensjahr nicht überschritten haben, werden gebeten, ihre wissenschaftlichen Arbeiten an den Sekretär der Deutschen DiabetesGesellschaft, Herrn Professor Dr. med. H. Ditschuneit, Zentrum für Innere Medizin der Universität Ulm, 79 Ulm/ Donau, Steinhövelstr. 9, unter Angabe von Anschrift und Lebenslauf bís spätestens 15. 2. 1971 einzusenden. Die Arbeiten sollten innerhalb des deutschen Sprachraumes entstanden sein. Der Lebenslauf soll den wissenschaftlichen Werdegang enthalten. - Die Verleihung des Preises erfolgt zu Beginn der 6. Tagung der Deutschen Diabetes-Gesellschaft am 14./15. Mai 1971 in Düsseldorf. Der Preisträger soll bei dieser Gelegenheit eine Bertram-Preis-Vorlesung halten, die über sein spezielles Arbeitsgebiet berichtet.

\section{Förderpreis der Deutschen Diabetes-Gesellschaft}

Für hervorragende Dissertationen auf dem Gebiet der Diabetologie wurde von den Firmen Bayer und Schering $\mathrm{AG}$ der ,Förderpreis der Deutschen Diabetes-Gesellschaft" ${ }^{\circ}$ 'estiftet. Er soll angehende Wissenschaftler auszeichnen und ihnen ermöglichen, sich an theoretischen Instituten des In- und Auslandes fortzubilden. Der Preis, der von der Deutschen Diabetes-Gesellschaft mit einer Urkunde verliehen wird, ist mit DM 11000, - dotiert, davon sind DM 10000 , für ein Stipendium bestimmt. - Deutschsprachige Bewerber werden gebeten, ihre Arbeit direkt mit der Promotions. urkunde oder - im Falle eines noch nicht abgeschlossenen Promotionsverfahrens - über den Doktorvater mit dem Referat über die Arbeit und einem Lebenslauf an den Vorsitzenden der Deutschen Diabetes-Gesellschaft, Herrn Prof. Dr. med. Karl Jahnke, Direktor der Medizinischen Klinik der Städt. Ferdinand-Sauerbruch-Krankenanstalten, 56 Wuppertal-Elberfeld bis spätestens 15. 2. 1971 einzureichen. Die Dissertation soll nicht älter als zwei Jahre sein und die Note „,sehr gut" erhalten haben. Mit dem Preis können auch solche in deutscher Sprache verfaßten Arbeiten ausgezeichnet werden, die den Dissertationen der deutschen Hochschulen entsprechen und an deutschsprachige Universitäten eingereicht wurden. - Die Verleihung des Preises erfolgt anläßlich des 6 . Kongresses der Deutschen Diabetes-Gesellschaft am 14./15. Mai 1971 in Düsseldorf.

\section{Corrigendum Notice}

Diabetologia 6, 373-378 (1970). H. Steiner and Chr. Hedinger, Storage of Acidophilic Granules in the Pituitary in Experimental Hyperglycaemia. Transpose legends to Figs. 4 and 5 on page 376 ; Figures are correct as printed.

Responsible for the text : Prof.Dr. K. Obendrsse, II. Med. Klinik u. Poliklinik der Universität, D-4000 Düsseldorf, Moorenstr. 5. Responsible for advertisements : EdGAR SeIDrER, D-1 Berlin 15, Kurfürstendamm 237. Springer-Verlag, Berlin, Heidelberg, New York. Printed in Germany by Druckerei Georg Appl, Wemding/Schwaben.

Copyright (C) by Springer-Verlag Berlin - Heidelberg 1970 